\title{
Online geocode in postal address using gps with synchronous database accessing
}

\author{
Mohammad Riyadh R. Sharba ${ }^{1}$, Hakim Adil Kadhim ${ }^{2}$, Salam A. W. Al-Abassi ${ }^{3}$, Nabeel Salih $\mathrm{Ali}^{4}$ \\ ${ }^{1,2,3}$ ECE Department, Faculty of Engineering, University of Kufa, Iraq \\ ${ }^{4}$ Information Technology Research and Development Centre, University of Kufa, Kufa, Najaf Governorate, Iraq
}

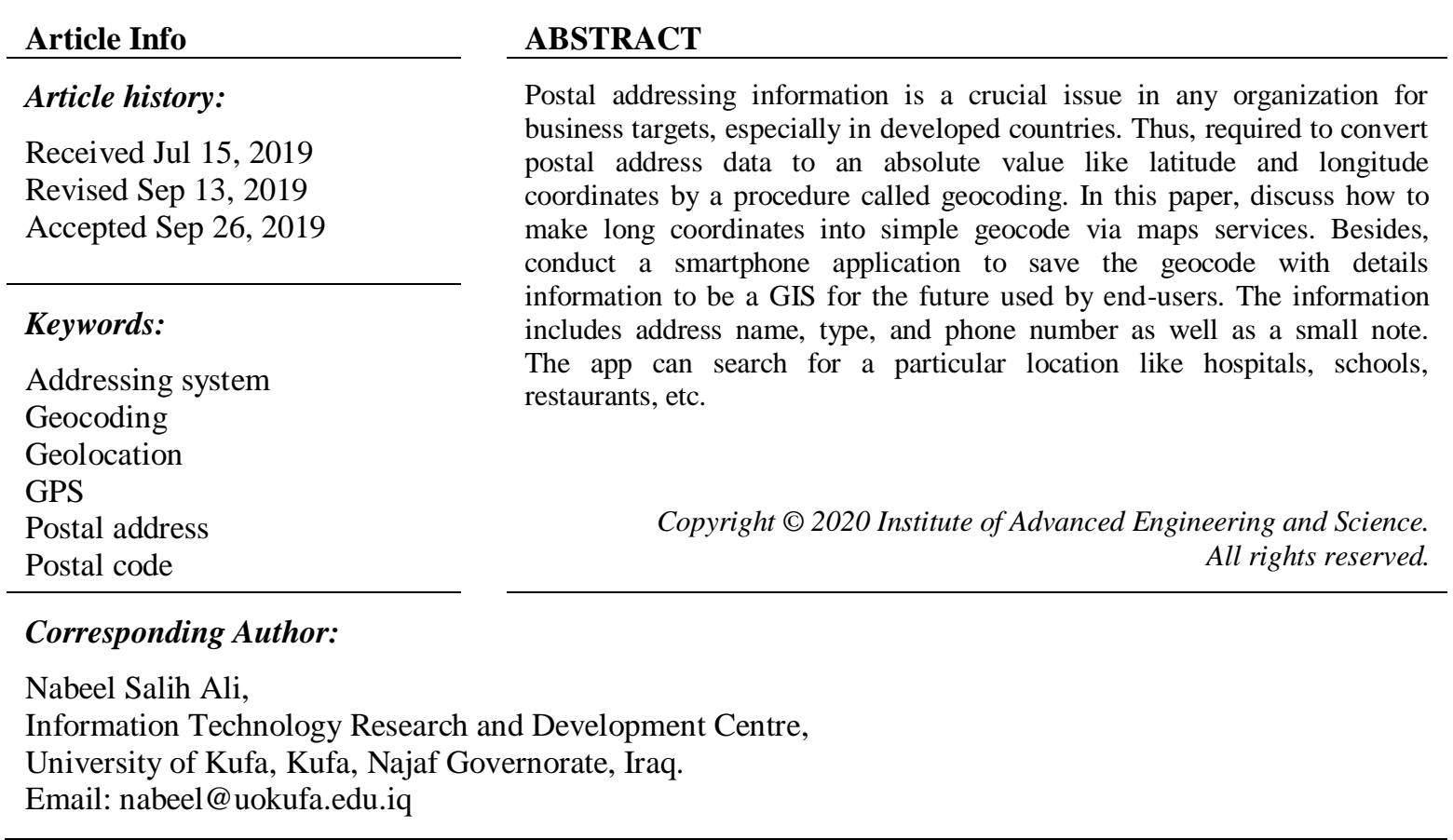

\section{INTRODUCTION}

In the internet era, huge data are produced, manipulate, and, consume and it was considered "one of the essential value-added pieces of information" among the advancement of the internet-based services [1-3]. Hence, maps and location-based services and applications are spread via widespread adoption recently [4-6]. Therefore, several devices that are used data with GPS, location tagging has become an increasingly prevalent practice [7-9]. These data contain over $80 \%$ a geographic reference as well GIS, and spatial analysis has adopted a technique in many different fields $[10,11]$. Thus, physical location data such as postal address require converted to absolute value, such as latitude and longitude coordinates by a procedure called geocoding $[12,13]$. A postal address is knowledge composition that encompasses different data such as suite information, street location, and geographical position $[14,15]$. Different countries have various postal addressing systems with diverse components ordering, formats, and components themselves [16]. Besides, postal address represents diverse languages and grammar in different countries. People are searching for a particular address by using a webpage with map services [17]. The postal address should be accurate, up-todate, easy-to-use, and applicable in online and international business processes [18]. Also, Postal codes are considered a unique geographic identifier used to study the population-based on assigning contextual or environmental information [19]. However, Geocoding is a technique used to assign geographic coordinates with a highly accurate way for an individual's residential location [20]. Postal codes are affected by Multifactor like delivery mode type, representative point type, and community size with observance to the accuracy of postal code spatial assignment [21]. Whether delivery mode type factor refers to a mode of postal delivery, representative point type factor points to the source of latitude-longitude coordinates consecutively [22]. 
Authors are attempts to produce several postal address and codes. According to [23], The authors proposed postal address detection from web pages. The approach experiments results were high precision (89.3\%) with (3.8\%) false alarms rate [23]. Likewise, the study in [24] is presented a geocoded postal address for location service-based; the conducted technique include MAC addresses and geographic position of wireless LAN access points, and it's resulted with high accuracy [24]. Besides, [25] proposed a geocoding method to design a rural address. The results have shown stability and easy to memorize of the address coding [21].

This paper discusses how to make simple, short geocode and use it as postal code in addressing maps service. Besides, the study designs a mobile-based application to implement the generated geocode with multi-functions. The smartphone app capable of inserting various information by a user himself, such as address name, type, and phone number and a small note. Later, the app provides the ability to search for a specific location like hospitals, schools, restaurants, etc. by providing a referenced database accessibility.

\section{MATERIALS AND METHODS}

To implement the proposed system, Software (S.W) and Hardware (H.W) materials are needed. These components are chosen as respect to multi-criteria, such as easy to use, cheap, and available. The H.W parts are Laptop HP Intel core i3, RAM 4G, Smartphone for testing like Samsung Galaxy J7. Whereas, the required S.W components are Microsoft visual studio community 2015, Xamarin environment with C\#, SQL Lite, Google maps API, XAML, and Jason. The study used Google Maps to offer satellite imagery road maps, $360^{\circ}$ all-encompassing perspectives of boulevards (Street View), constant movement conditions (Google Traffic), and course making arrangements for going by foot, auto, bike (in beta), or accessible transportation. Also, Google Maps provide API, while used Xamarin environment which is a cross-platform app for a $\mathrm{C \#}$ programming language to implement the system in smartphones OS. Figure 1 describes a block diagram of the proposed method.

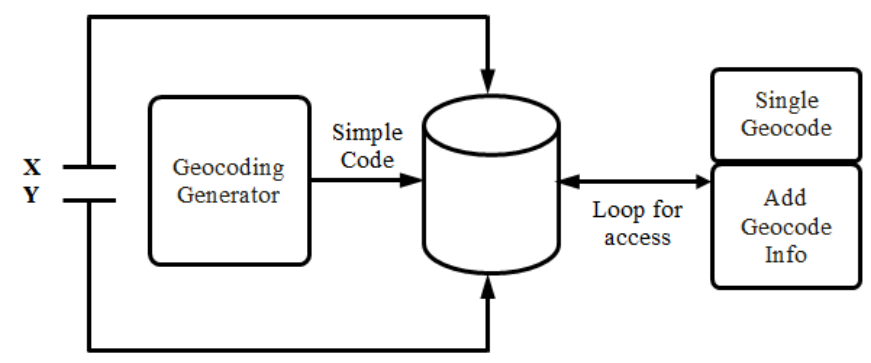

Figure 1. Block diagram of the proposed system

\section{RESULTS AND DISCUSSIONS}

Several steps can be achieved to implement the introduce system such as:

Step 1: The application uses the google maps API to import the map to the application as a workspace.

Step 2: We build the application depend on the latitude and the longitude of the map to generate a unique code (Geocoding) as Figure 2 shows how these lines cross a specific point with a unique value.

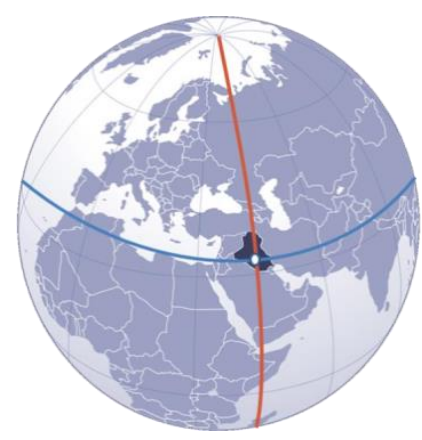

Figure 2. Point of the earth (coordinates) 
Step 3: The proposing algorithm based on converting between two different based number, such as converting between decimal to hexadecimal. The difference is that the algorithm converts the longitude and the latitude numbers (base to a value of 10) to a new format based on the value of 62. This base vale (62) is comes from:

(26) English Alphabetics + (26) English Capital Alphabetics + (10) integer from 0 - 9

It means that this system uses 62 values to represent a number. The procedure of the algorithm is proposed as follow:

Algorithm procedures for converting between two different based numbers as shown in Figure 3. Figure 4 describes the pseudo-code of the converting the longitude and latitude values to a new base value.

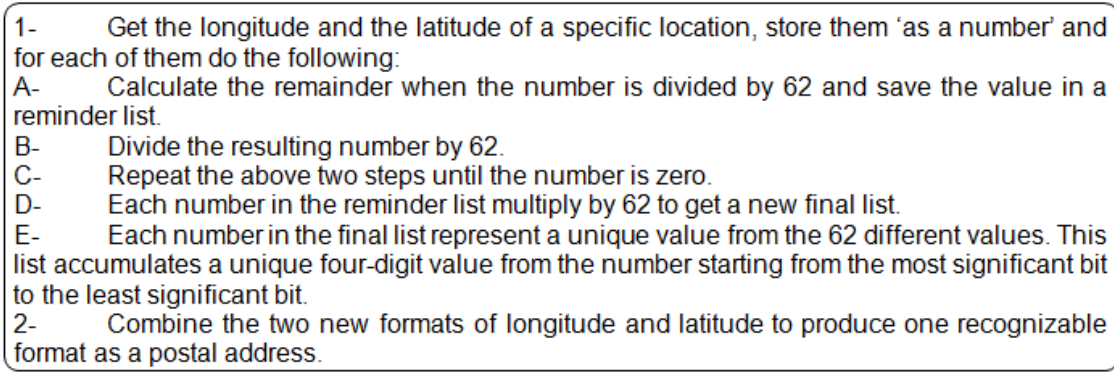

Figure 3. Algorithm procedures for converting between two different based numbers

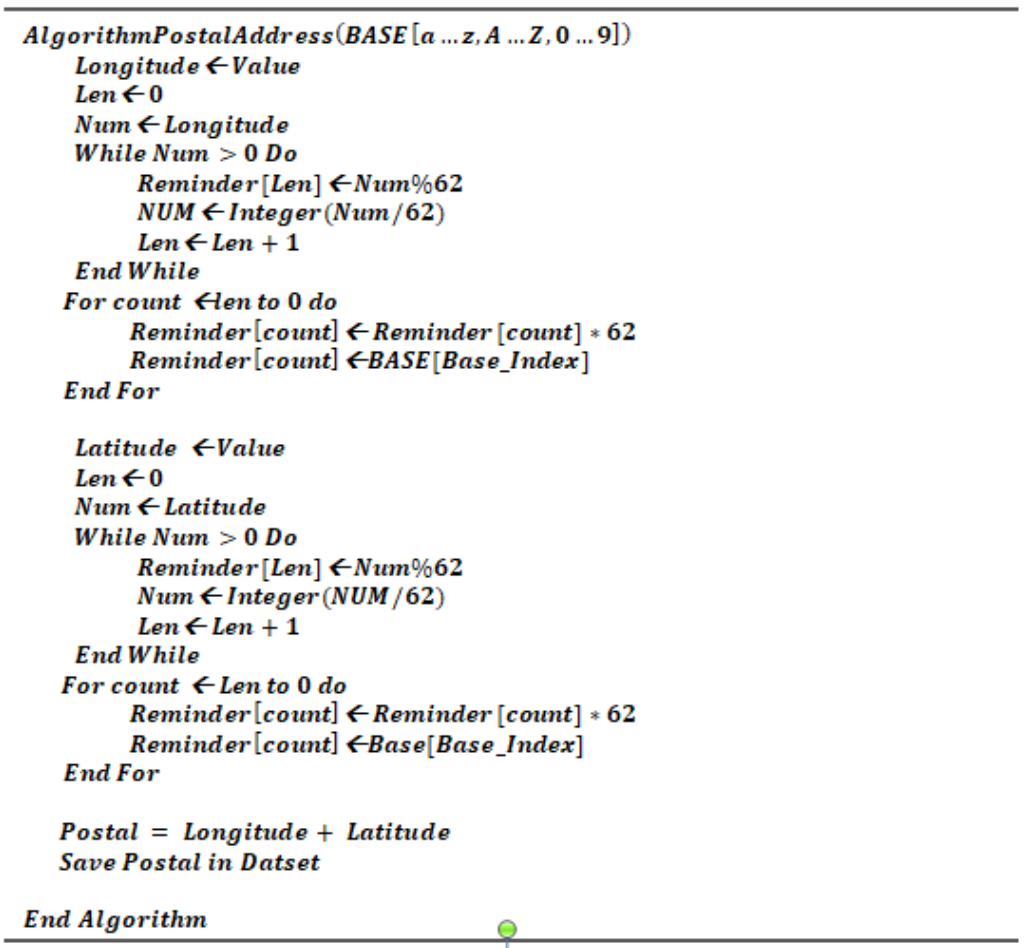

Figure 4. Longitude and Latitude to postal address

Based on Figure 1, let Assume the selected point is $32.081737,44.395168$ coordinates which have the different properties like; very long and complicated, difficult to use and keep in mind, and hard to share. Thus, the proposed procedure in the implemented application solve these issues in the code. This android app depending on the latitude and the longitude of the particular point to generate a unique code. The point which the system selected will be (29ATm.2cBbc6) as shown in Figure 5.

Online geocode in postal address using gps with synchronous database... (Mohammad Riyadh R. Sharba) 


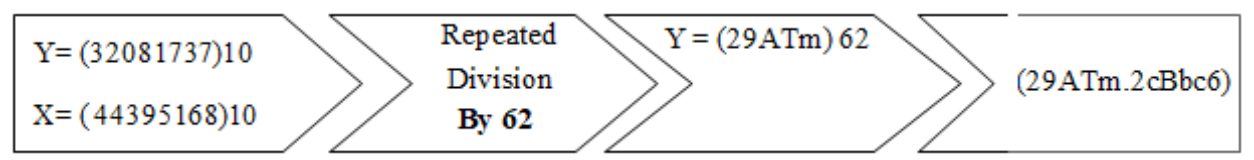

Figure 5. Geocoding generator method

On the other hands, our postal code can be easily backed as a multiplication of longitude and latitude values. As the 62 base values $[0,1,2,3,4,5,6,7,8,9, \mathrm{a}, \mathrm{b}, \mathrm{c}, \mathrm{d}, \mathrm{e}, \mathrm{f}, \mathrm{g}, \mathrm{h}, \mathrm{I}, \mathrm{j}, \mathrm{k}, \mathrm{l}, \mathrm{m}, \mathrm{n}, \mathrm{o}, \mathrm{p}, \mathrm{q}, \mathrm{u}, \mathrm{r}$, s, t, v, w, x, y, z, A, B, C, D, E, F, G, H, I, J, K, L, M, N, O, P, Q, U, R, S, T, V, W, X, Y, Z] are already categorized based on integer numbers, English alphabetic and capital English alphabetic, all we need is to prefetch any postal code to its original number. The process starts from the right (most significant digit) of the postal code to the left. Firstly, each digit is recategorized according to the 62 values. Then it multiplies with the proper base (power of 62). Finally, the result of each digit is added up the produce the final number. Figure 6 shows an example of fetching (29Atm) to the real longitude value.

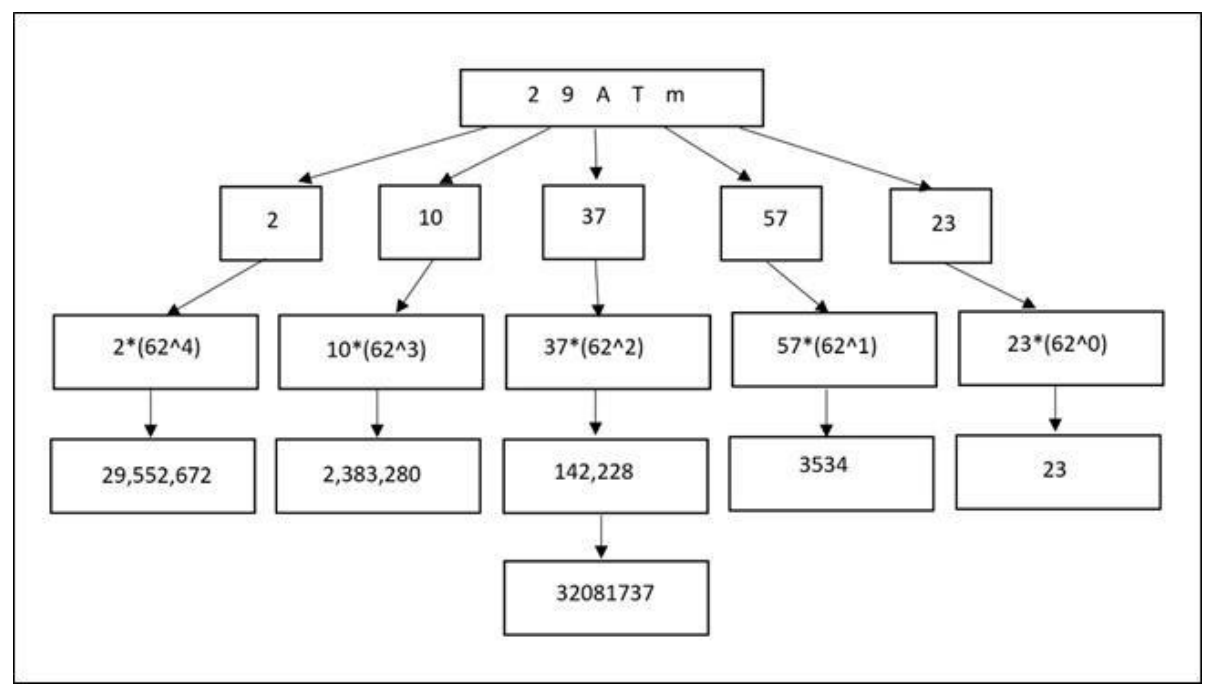

Figure 1. Procedure of fetching unique code to its original value

Step 4: We connect the application with a local database (SQL Late) to store the user data. Step 5: Jason is used to transfeing data between SQL Late and the phone.

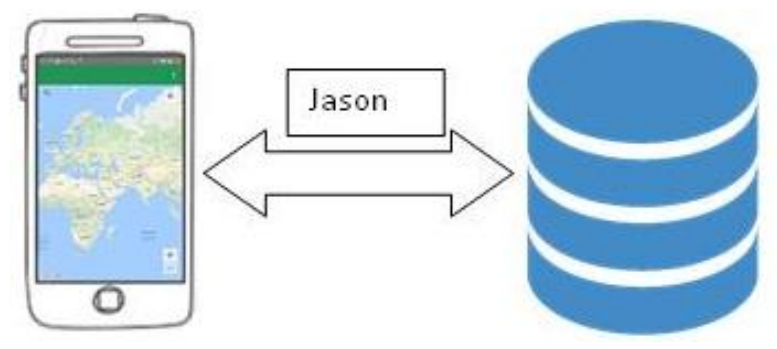

Figure 7. Data transmitting between user and phone and reference database

Step 6: The interfaces of the application designed by the XAML.

Step 7: The application can track the user location using GPS on the google maps API. Figure 8 provides the conducted algorithm and procedures of the application. 


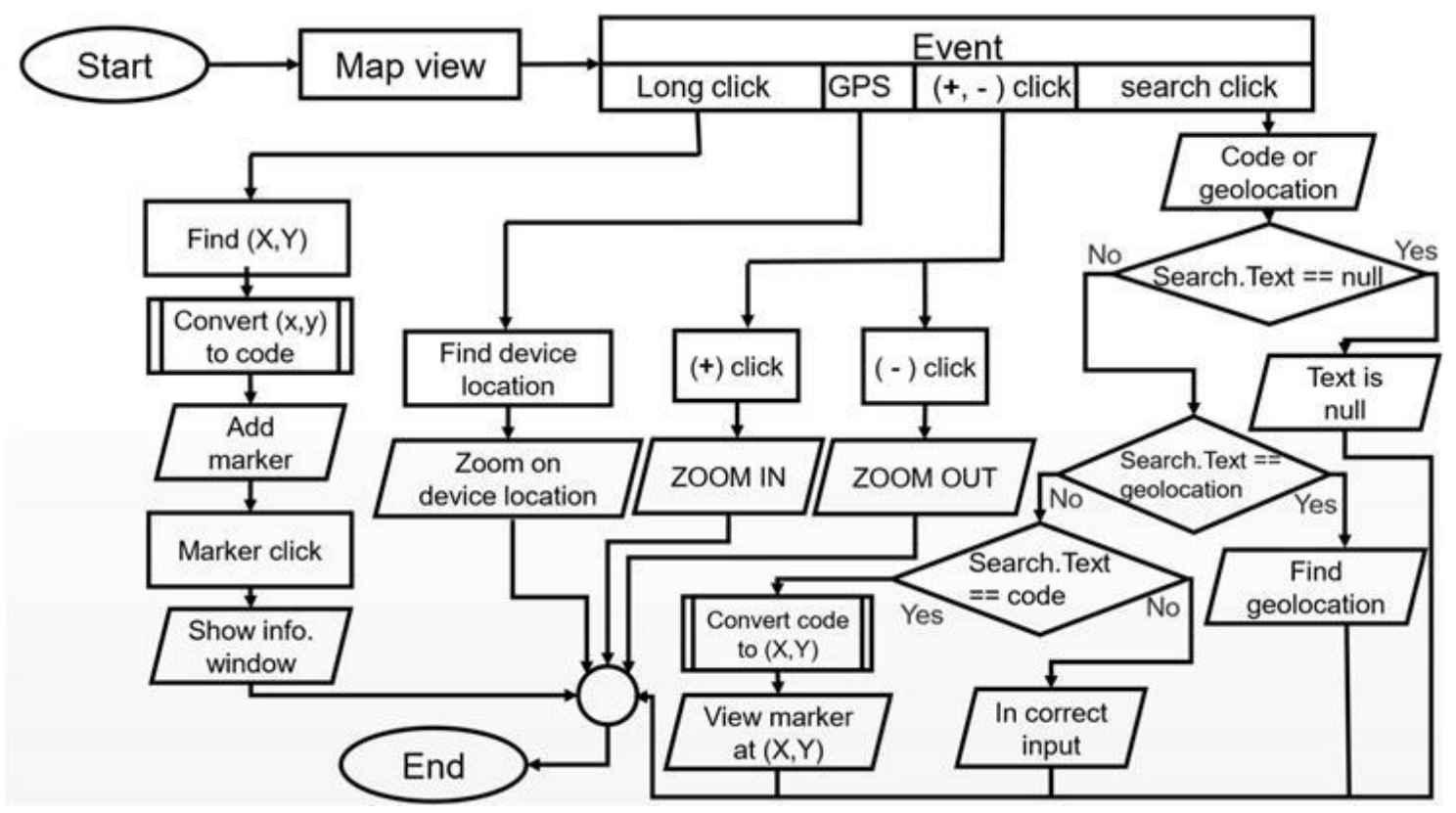

Figure 8. Algorithm of the smartphone application

We convert every point in the earth to small code between 2-8 char. It consists of numbers, capital, and small letters in Figure 10. Also, we add the ability to the user to uploading the required information for the particular address as we see in Figure 9. So, the presented application will give a location information service to the user. Whereas, the user capable of search about a specific place or location in the particular map via its geolocation and the code place (see Figure 10).

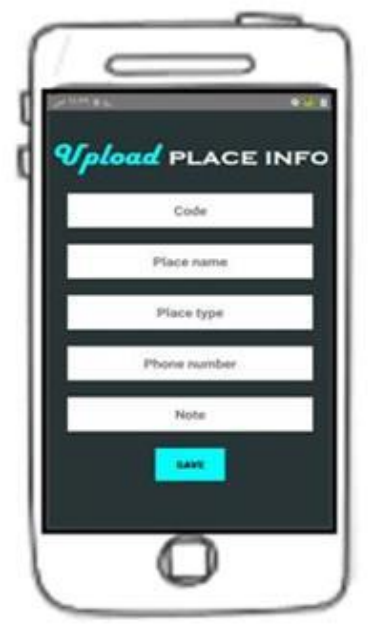

Figure 9. Geocode Information Insertion by User

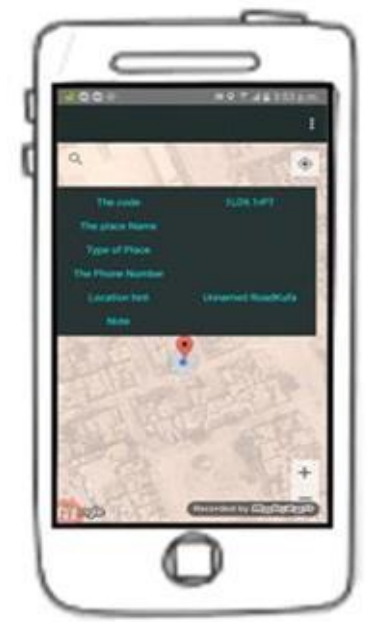

Figure 10. Extract the geocode information from DB reference

\section{CONCLUSIONS AND FUTURE WORK}

In this study, proposed an application to generate a GIS from simple geocode based on GPS and reference database. The system provides the ability to most straight forward the long coordinate's code to simple geocode which the code depends on longitude and latitude hence it has various advantages such as itis unique code, covered all the world the application is scalable among the smart devices. In the future, it is expected to be used in a wide range at the future via enabling the user to update the place, minimize the number of code digits, and avoid the lower-case letters, working on the application design and performance.

Online geocode in postal address using gps with synchronous database... (Mohammad Riyadh R. Sharba) 


\section{REFERENCES}

[1] Roongpiboonsopit, D., \& Karimi, H. A. (2010). "Comparative evaluation and analysis of online geocoding services." International Journal of Geographical Information Science, 24(7), 1081-1100.

[2] Alharan, A. F., Fatlawi, H. K., \& Ali, N. S. (2019). "A cluster-based feature selection method for image texture classification." Indonesian Journal of Electrical Engineering and Computer Science, 14(3), 1433-1442.

[3] B. Xue, et al., "A survey on evolutionary computation approaches to feature selection," IEEE Transactions on Evolutionary Computation, vol. 20, pp. 606-626, 2016.

[4] Goldberg, D. W., Wilson, J. P., \& Knoblock, C. A. (2007). From text to geographic coordinates: the current state of geocoding. URISA-WASHINGTON DC-, 19(1), 33.

[5] Kadhim, H. A., Ali, N. S., \& Dheyaa, M. A. (2019). "Management and Achieving System for Metal Detection Robot Using Wireless-Based Technology and Online Database Registry." International Journal of Power Electronics and Drive System, 10(1), 219-229.

[6] Ali, N. S., \& Alyasseri, Z. A. A. (2017). Wireless Sensor Network and Web Application Hybrid Scheme for Healthcare Monitoring. Journal of Soft Computing and Decision Support Systems, 4(5), 1-7.

[7] Ali, N. S., Kadhim, H. A., \& Abdulsahib, D. M. (2019). "Multi-function intelligent robotic in metals detection applications." TELKOMNIKA, 17(4), 2058-2069.

[8] Rjeib, H. D., Ali, N. S., Al Farawn, A., Al-Sadawi, B., \& Alsharqi, H. (2018). Attendance and information system using rfid and web-based application for academic sector. International Journal of Advanced Computer Science and Applications, 9(1).

[9] Ali, N. S., Alyasseri, Z. A. A., \& Abdulmohson, A. (2018). Real-Time Heart Pulse Monitoring Technique Using Wireless Sensor Network and Mobile Application. International Journal of Electrical and Computer Engineering, $8(6), 5118$.

[10] Esri, 2014. Reveal More Value in Your Data with Location Analytics. Available at http://www.esri.com/library/whitepapers/pdfs/reveal- more-value.pdf

[11] Zandbergen, P. A. (2008). A comparison of address point, parcel and street geocoding techniques. Computers, Environment and Urban Systems, 32(3), 214-232.

[12] Krieger, N., Waterman, P., Lemieux, K., Zierler, S., \& Hogan, J. W. (2001). "On the wrong side of the tracts? Evaluating the accuracy of geocoding in public health research." American journal of public health, 91(7), 1114.

[13] Fernandez, S. G., Palanisamy, R., \& Vijayakumar, K. (2018). "GPS \& GSM Based Accident Detection And Auto Intimation." Indonesian Journal of Electrical Engineering and Computer Science, 11(1), 336-361.

[14] Cai, W., Wang, S., \& Jiang, Q. (2005, March). Address extraction: Extraction of location-based information from the web. In Asia-Pacific Web Conference (pp. 925-937). Springer, Berlin, Heidelberg.

[15] Sanzgiri, S. M., Gilliland, P. C., Harokopus, W. P., \& Svigelj, J. (1999). U.S. Patent No. 5,923,296. Washington, DC: U.S. Patent and Trademark Office.

[16] Mulasastra, I., \& Taplaksint, A. (2015, December). "Elementization of Thai postal addresses: A hybrid approach." In Electrical and Computer Engineering (WIECON-ECE), 2015 IEEE International WIE Conference on (pp. 561564). IEEE.

[17] Chang, C. H., \& Li, S. Y. (2010, August). "MapMarker: Extraction of postal addresses and associated information for general web pages". In Web Intelligence and Intelligent Agent Technology (WI-IAT), 2010 IEEE/WIC/ACM International Conference on (Vol. 1, pp. 105-111). IEEE.

[18] Trninic, D. (2006). "The Semantic Web-Ready Postal Address Management in Global E-Commerce." BLED 2006 Proceedings, 16.

[19] Khan, S., Pinault, L., Tjepkema, M., \& Wilkins, R. (2018). Positional accuracy of geocoding from residential postal codes versus full street addresses. Health reports, 29(2), 3-9.

[20] Mazumdar, S., Rushton, G., Smith, B. J., Zimmerman, D. L., \& Donham, K. J. (2008). Geocoding accuracy and the recovery of relationships between environmental exposures and health. International Journal of Health Geographics, 7(1), 13.

[21] DeLuca, P. F., \& Kanaroglou, P. S. (2008). Effects of alternative point pattern geocoding procedures on first and second order statistical measures. Journal of Spatial Science, 53(1), 131-141.

[22] Bonner, M. R., Han, D., Nie, J., Rogerson, P., Vena, J. E., \& Freudenheim, J. L. (2003). Positional accuracy of geocoded addresses in epidemiologic research. Epidemiology, 14(4), 408-412.

[23] Can, L., Qian, Z., Xiaofeng, M., \& Wenyin, L. (2005, April). Postal address detection fromweb documents. In null (pp. 40-45). IEEE.

[24] Chandrasekaran, G., Ergin, M. A., Gruteser, M., \& Martin, R. P. (2007, September). Bootstrapping a location service through geocoded postal addresses. In International Symposium on Location-and Context-Awareness (pp. 1-16). Springer, Berlin, Heidelberg.

[25] Pan, Y., Chen, B., Lu, Z., Li, S., Zhang, J., \& Zhou, Y. (2010, November). An address geocoding method for improving rural spatial information infrastructure. In Sixth International Symposium on Digital Earth: Models, Algorithms, and Virtual Reality (Vol. 7840, p. 784006). International Society for Optics and Photonics. 